\title{
Binding Energies and Dissociation Temperatures of Heavy Quarkonia at Finite Temperature and Chemical Potential in the $N$-Dimensional Space
}

\author{
M. Abu-Shady (D), ${ }^{1}$ T. A. Abdel-Karim, ${ }^{1}$ and E. M. Khokha ${ }^{2}$ \\ ${ }^{1}$ Department of Applied Mathematics, Faculty of Science, Menoufia University, Shibin El Kom, Egypt \\ ${ }^{2}$ Department of Basic Science, Modern Academy of Engineering and Technology, Cairo, Egypt \\ Correspondence should be addressed to M. Abu-Shady; dr.abushady@gmail.com
}

Received 23 July 2017; Accepted 29 November 2017; Published 8 January 2018

Academic Editor: Juan José Sanz-Cillero

Copyright (c) 2018 M. Abu-Shady et al. This is an open access article distributed under the Creative Commons Attribution License, which permits unrestricted use, distribution, and reproduction in any medium, provided the original work is properly cited. The publication of this article was funded by $\mathrm{SCOAP}^{3}$.

\begin{abstract}
The $N$-dimensional radial Schrödinger equation has been solved using the analytical exact iteration method (AEIM), in which the Cornell potential is generalized to finite temperature and chemical potential. The energy eigenvalues have been calculated in the $\mathrm{N}$-dimensional space for any state. The present results have been applied for studying quarkonium properties such as charmonium and bottomonium masses at finite temperature and quark chemical potential. The binding energies and the mass spectra of heavy quarkonia are studied in the $N$-dimensional space. The dissociation temperatures for different states of heavy quarkonia are calculated in the three-dimensional space. The influence of dimensionality number $(N)$ has been discussed on the dissociation temperatures. In addition, the energy eigenvalues are only valid for nonzero temperature at any value of quark chemical potential. A comparison is studied with other recent works. We conclude that the AEIM succeeds in predicting the heavy quarkonium at finite temperature and quark chemical potential in comparison with recent works.
\end{abstract}

\section{Introduction}

The solution of the radial Schrödinger equation with spherically symmetric potentials has vital applications in different fields of physics such as atoms, molecules, hadronic spectroscopy, and high energy physics. The Schrödinger equation has been solved by operator algebraic method [1], power series method [2,3], and path integral method [4], in addition to quasi-linearization method (QLM) [5], point canonical transformation (PCT) [6], Hill determinant method [7], and the conventional series solution method [8]. Recently, most of the theoretical studies have been developed to study the solutions of radial Schrödinger equation in the higher dimensions. These studies are general and one can directly obtain the results in the lower dimensions [9-23]. The $N$-dimensional Schrödinger equation has been solved by various methods as the Nikiforov-Uvarov (NU) method [912], asymptotic iteration method (AIM) [13], Laplace Transform method $[14,15]$, supersymmetric quantum mechanics
(SUSQM) [16], power series technique [17], Pekeris type approximation [18], and the analytical exact iteration method (AEIM) [19].

The $N$-dimensional radial Schrödinger equation has been solved for different types of spherical symmetric potentials as Coulomb potential [15], pseudo-harmonic potential [20], Mie-type potential [21], energy-dependent potential [11], Kratzer potential [22], and Cornell potential type [13, 23] that consists of the Coulomb term and the linear term, anharmonic potential [14], the Cornell potential with harmonic oscillator potential [12], and the extended Cornell potential [19].

The solution of Schrödinger equation has been used in different studies to describe the properties of heavyquarkonium systems at finite temperature. Many efforts have been devoted to calculating the mass spectra of charmonium and bottomonium mesons and determining the binding energy and the dissociation temperatures of heavy quarkonia. In [24, 25], the authors have calculated the 
dissociation rates of quarkonium ground states by tunneling and direct thermal activation to the continuum and the binding energies and scattering phase shifts for the lowest eigenstates in the charmonium and bottomonium systems in hot gluon plasma. In $[26,27]$, the deconfinement and properties of the resulting quark-gluon plasma (QGP) have been investigated by studying the medium behavior of heavyquark bound states in statistical quantum chromodynamics and the spectral analysis of quarkonium states in a hot medium of deconfined quarks and gluons and the thermal properties of QGP are discussed. In [28-30], the authors have solved the Schrödinger equation at finite temperature for the charmonium and bottomonium states by employing an effective temperature dependent potential given by a linear combination of the color singlet free and internal energies and discussed the quarkonium spectral functions in a quarkgluon plasma. The dissociation of quarkonia has been studied by correcting the full Cornell potential through the hard-loop resumed gluon propagator and the hard thermal loop (HTL) approximation [31,32]. Moreover, the binding energies of the heavy quarkonia states are studied in detail in $[33,34]$.

At finite temperature and chemical potential, Vija and Thoma [35] have extended the effective perturbation theory for gauge theories at finite temperature and chemical potential for studying the collisional energy loss of heavy quarks in QGP. In [36, 37], the authors have generalized a thermodynamic quasi-particle description of deconfined matter to finite chemical potential and analyzed the response of color singlet and color averaged heavy-quark free energies to a nonvanishing baryon chemical potential. On the same hand, the effect of chemical potential is studied on the photon production of quantum chromodynamics (QCD) plasma, dissipative hydrodynamic effects on QGP, and thermodynamic properties of the QGP [38-42] by using different methods. At finite chemical potential and small temperature region, the dissociation of quarkonia states has been studied in a deconfined medium of quarks and gluons in [43].

The aim of this work is to find the analytic solution of the $\mathrm{N}$-dimensional radial Schrödinger equation with generalized Cornell potential at finite temperature and chemical potential using the analytical exact iteration method (AEIM) to obtain the energy eigenvalues, where the energy eigenvalues are only valid for nonzero temperature for any value of quark chemical potential. So far no attempt has been made to solve the $N$ dimensional radial Schrödinger when finite temperature and chemical potential are included by using AEIM. In addition, the application of present results on quarkonium properties has been investigated such as the mass spectra of heavy quarkonium and the dissociation temperature for different states of heavy quarkonia. The influence of the dimensionality number, which is not considered in many recent works, has been investigated on the binding energy and the dissociation temperature at finite temperature and chemical potential.

The paper is organized as follows: the background of the study of previous efforts is introduced in Section 1. In Section 2, the analytic solution of the $\mathrm{N}$-dimensional radial Schrödinger equation is derived. In Section 3, the results are discussed. In Section 4, summary and conclusion are presented.

\section{Analytic Solution of the $N$-Dimensional Radial Schrödinger Equation with the Cornell Potential at Finite Temperature and Chemical Potential}

The $\mathrm{N}$-dimensional radial Schrödinger equation for two particles interacting via a spherically symmetric potential takes the following form $[14,44]$ :

$$
\begin{aligned}
& {\left[\frac{d^{2}}{d r^{2}}+\frac{N-1}{r} \frac{d}{d r}-\frac{l(l+N-2)}{r^{2}}\right.} \\
& \left.+2 \mu_{\mathrm{QQ}}\left(E_{n l}-U(r)\right)\right] \psi(r)=0,
\end{aligned}
$$

where $l, N$, and $\mu_{\mathrm{QQ}}$ are the angular quantum number, the dimensional number, and reduced mass of the two particles $\mu_{\bar{Q} \bar{Q}}=m_{\mathrm{Q}} m_{\bar{Q}} /\left(m_{\mathrm{Q}}+m_{\overline{\mathrm{Q}}}\right)$, respectively.

Inserting $\psi(r)=R(r) / r^{(N-1) / 2}$ in (1), we obtain

$$
\left[\frac{d^{2}}{d r^{2}}-\frac{\lambda^{2}-1 / 4}{r^{2}}+2 \mu_{\mathrm{QQ}}\left(E_{n l}-U(r)\right)\right] R(r)=0,
$$

with $\lambda=l+(N-2) / 2$, where $U(r)$ is the Cornell potential that takes the following form [45]:

$$
U(r)=\sigma r-\frac{\alpha_{s}}{r},
$$

with $\sigma=0.192 \mathrm{GeV}^{2}$ and $\alpha_{s}=0.471$. The potential is modified in QGP to study the binding energy and dissociation temperature by including Debye screening mass as follows $[45,46]$ :

$$
U\left(r, m_{D}\right)=\frac{\sigma}{m_{D}}\left(1-e^{-m_{D}(T, \mu) r}\right)-\frac{\alpha_{s}}{r} e^{-m_{D}(T, \mu) r},
$$

where $m_{D}(T, \mu)$ is the Debye screening mass at finite temperature and quark chemical potential [37]:

$$
\begin{aligned}
& m_{D}(T, \mu) \\
& \quad=g(T) T \sqrt{\frac{N_{c}}{3}+\frac{N_{f}}{6}} \sqrt{1+\frac{3 N_{f}}{\left(2 N_{c}+N_{f}\right) \pi^{2}}\left(\frac{\mu}{T}\right)^{2}},
\end{aligned}
$$

where $N_{f}$ is the number of quark flavors, $N_{c}$ is the number of colors, and $g(T)$ is the QCD coupling constant at finite temperature [47]:

$$
g(T)=\frac{1}{\left(11 N_{c}-2 N_{f}\right) \log \left(T^{2} / \Lambda_{\mathrm{QCD}}^{2}\right)} .
$$

Using $e^{-m_{D}(T, \mu) r}=\sum_{k=0}^{\infty}\left(-m_{D}(T, \mu) r\right)^{k} / k$ ! in (4) with neglecting the higher orders at $m_{D}(T, \mu) r \ll 1$, thus (4) takes the following form:

$$
V(r)=-a r^{2}+b r+c-\frac{d}{r},
$$

where 


$$
\begin{aligned}
& a=\frac{1}{2} \sigma m_{D}(T, \mu), \\
& b=\frac{1}{2}\left(2 \sigma-\alpha_{s} m_{D}(T, \mu)^{2}\right), \\
& c=\alpha_{s} m_{D}(T, \mu), \\
& d=\alpha_{s} .
\end{aligned}
$$

Substituting (7) into (2), we obtain

$$
\begin{aligned}
& R^{\prime \prime}(r) \\
& \quad=\left[-\varepsilon_{n l}+a_{1} r^{2}+b_{1} r+c_{1}-\frac{d_{1}}{r}+\frac{\lambda^{2}-1 / 4}{r^{2}}\right] R(r),
\end{aligned}
$$

where

$$
\begin{aligned}
& \varepsilon_{n l}=2 \mu_{\mathrm{QQ}} E_{n l}, \\
& a_{1}=\left|-2 \mu_{\mathrm{QQ}} a\right|, \\
& b_{1}=2 \mu_{\mathrm{QQ}} b, \\
& c_{1}=2 \mu_{\mathrm{QQ}} c, \\
& d_{1}=2 \mu_{\mathrm{QQ}} d .
\end{aligned}
$$

The analytical exact iteration method (AEIM) requires the following ansatz for the wave function as in [48-50]:

$$
R(r)=f_{n}(r) \exp \left[g_{l}(r)\right],
$$

where

$$
\begin{aligned}
& f_{n}(r)= \begin{cases}1, & n=0 \\
\prod_{i=1}^{n}\left(r-\alpha_{i}^{(n)}\right) & n=1,2,3, \ldots,\end{cases} \\
& g_{l}(r)=-\frac{1}{2} \alpha r^{2}-\beta r+\delta \ln r, \quad \alpha>0, \quad \beta>0 .
\end{aligned}
$$

From (11), we obtain

$$
\begin{aligned}
& R_{n l}^{\prime \prime}(r)=\left(g_{l}^{\prime \prime}(r)+g_{l}^{\prime 2}(r)+\frac{f_{n}^{\prime \prime}(r)+2 g_{l}^{\prime}(r) f_{n}^{\prime}(r)}{f_{n}(r)}\right) \\
& \cdot R_{n l}(r) .
\end{aligned}
$$

By comparing (9) and (14), we obtain

$$
\begin{aligned}
& a_{1} r^{2}+b_{1} r+c_{1}-\frac{d_{1}}{r}+\frac{\lambda^{2}-1 / 4}{r^{2}}-\varepsilon_{n l} \\
& =g_{l}^{\prime \prime}(r)+g_{l}^{\prime 2}(r)+\frac{f_{n}^{\prime \prime}(r)+2 g_{l}^{\prime}(r) f_{n}^{\prime}(r)}{f_{n}(r)} .
\end{aligned}
$$

At $(n=0)$, substituting (12) and (13) into (15) gives

$$
\begin{gathered}
a_{1} r^{2}+b_{1} r+c_{1}-\frac{d_{1}}{r}+\frac{\lambda^{2}-1 / 4}{r^{2}}-\varepsilon_{0 l} \\
=\alpha^{2} r^{2}+2 \alpha \beta r-\alpha[1+2(\delta)]+\beta^{2}-\frac{2 \beta \delta}{r} \\
+\frac{\delta(\delta-1)}{r^{2}} .
\end{gathered}
$$

By comparing the corresponding powers of $r$ on both sides of (16), one obtains

$$
\begin{aligned}
\alpha & =\sqrt{a_{1}}, \\
\beta & =\frac{b_{1}}{2 \sqrt{a_{1}}}, \\
d_{1} & =2 \beta \delta,
\end{aligned}
$$

$$
\begin{aligned}
\delta(\delta+1) & =\lambda^{2}-\frac{1}{4} \Longrightarrow \\
\delta & =\frac{1}{2}(1 \pm 2 \lambda), \\
\varepsilon_{0 l} & =\alpha[1+2(\delta)]+c_{1}-\beta^{2} .
\end{aligned}
$$

From (17a)-(17e) and (10), by taking the positive sign in (17d), then the ground state energy is

$$
E_{0 l}=\sqrt{\frac{a}{2 \mu_{\mathrm{QQ}}}}(N+2 l)+c-\frac{b^{2}}{4 a} .
$$

For the first node $(n=1)$, we use the functions $f_{1}(r)=(r-$ $\left.\alpha_{1}^{(1)}\right)$ and $g_{l}(r)$ from (13). Equation (15) takes the following form:

$$
\begin{aligned}
a_{1} r^{2}+ & b_{1} r+c_{1}-\frac{d_{1}}{r}+\frac{\lambda^{2}-1 / 4}{r^{2}}-\varepsilon_{1 l} \\
= & \alpha^{2} r^{2}+2 \alpha \beta r-\alpha[1+2(\delta+1)]+\beta^{2} \\
& -\frac{2\left[\beta(\delta+1)+\alpha \alpha_{1}^{(1)}\right]}{r}+\frac{\delta(\delta-1)}{r^{2}} .
\end{aligned}
$$

Then, the relations between the parameters of the potential and the coefficients $\alpha, \beta, \delta$, and $\alpha_{1}^{(1)}$ are given by

$$
\begin{aligned}
\alpha & =\sqrt{a_{1}}, \\
\beta & =\frac{b_{1}}{2 \sqrt{a_{1}}}, \\
d_{1} & =2 \beta(\delta+1), \\
\delta & =\frac{1}{2}(1 \pm 2 \lambda), \\
\varepsilon_{1 l} & =\alpha[1+2(\delta+1)]+c_{1}-\beta^{2}, \\
d_{1}-2 \beta(\delta+1) & =2 \alpha \alpha_{1}^{(1)}, \\
\left(d_{1}-2 \beta \delta\right) \alpha_{1}^{(1)} & =2 \delta .
\end{aligned}
$$

Using (20a)-(20g) and (10), we obtain the formula $E_{1 l}$ as

$$
E_{1 l}=\sqrt{\frac{a}{2 \mu_{\mathrm{QQ}}}}(N+2 l+2)+c-\frac{b^{2}}{4 a} .
$$



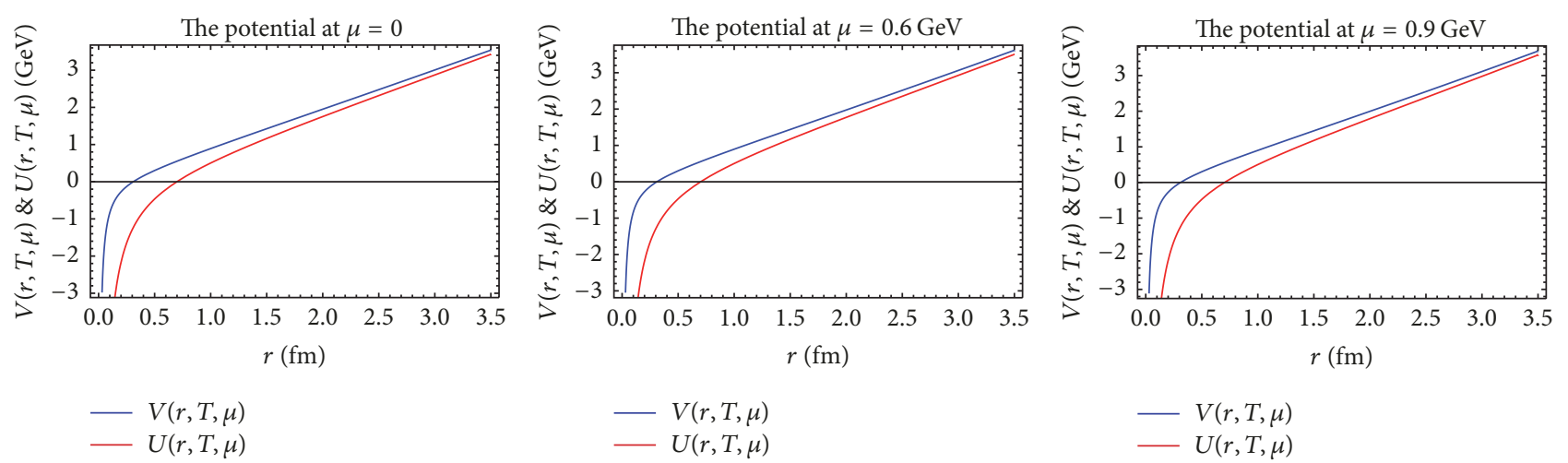

Figure 1: The comparison between the two potentials. The red line is the exact potential $U(r, T, \mu)$ and the blue line is the approximate potential $V(r, T, \mu)$ for different values of chemical potential at $T=250 \mathrm{MeV}$.

For the second node $(n=2)$, we use $f_{2}(r)=\left(r-\alpha_{1}^{(2)}\right)\left(r-\alpha_{2}^{(2)}\right)$ and $g_{l}(r)$ from (13) to solve (15) which gives

$$
\begin{aligned}
a_{1} r^{2} & +b_{1} r+c_{1}-\frac{d_{1}}{r}+\frac{\lambda^{2}-1 / 4}{r^{2}}-\varepsilon_{2 l} \\
= & \alpha^{2} r^{2}+2 \alpha \beta r-\alpha[1+2(\delta+2)]+\beta^{2} \\
& \quad-\frac{2\left[\beta(\delta+2)+\alpha\left(\alpha_{1}^{(2)}+\alpha_{2}^{(2)}\right)\right]}{r}+\frac{\delta(\delta-1)}{r^{2}} .
\end{aligned}
$$

Thus, the relations between the coefficients $\alpha, \beta, \delta, \alpha_{1}^{(2)}$, and $\alpha_{2}^{(2)}$ are given by

$$
\begin{aligned}
& \alpha=\sqrt{a_{1}}, \\
& \beta=\frac{b_{1}}{2 \sqrt{a_{1}}}, \\
& \delta=\frac{1}{2}(1 \pm 2 \lambda), \\
& \varepsilon_{2 l}=\alpha[1+2(\delta+2)]+c_{1}-\beta^{2}, \\
& d_{1}-2 \beta(\delta+2)=2 \alpha\left(\alpha_{1}^{(2)}+\alpha_{2}^{(2)}\right) \text {, } \\
& \left(d_{1}-2 \beta \delta\right) \alpha_{1}^{(2)} \alpha_{2}^{(2)}=2 \delta\left(\alpha_{1}^{(2)}+\alpha_{2}^{(2)}\right), \\
& {\left[d_{1}-2 \beta(\delta+1)\right]\left(\alpha_{1}^{(2)}+\alpha_{2}^{(2)}\right)} \\
& =4 \alpha\left(\alpha_{1}^{(2)} \alpha_{2}^{(2)}\right)+2(2 \delta+1) \text {. }
\end{aligned}
$$

Hence, the formula $E_{2 l}$ is given by

$$
E_{2 l}=\sqrt{\frac{a}{2 \mu_{\mathrm{QQ}}}}(N+2 l+4)+c-\frac{b^{2}}{4 a} .
$$

Then, the iteration method is repeated many times. Therefore, the exact energy formula for any state in the $N$-dimensional space is written as

$$
\begin{aligned}
E_{n l}^{N}=\sqrt{\frac{a}{2 \mu_{\mathrm{QQ}}}}(N+2 l+2 n)+c-\frac{b^{2}}{4 a}, & \\
& n=0,1,2, \ldots .
\end{aligned}
$$

According to (8), we note that (25) is only valid for finite temperature, since, at $T=0$, the parameter $a$ equals zero. Therefore, the energy eigenvalues diverge at this case.

\section{Discussion of Results}

In the first part of this section, we compare between the exact potential $U(r, T, \mu)$ in (4) and the approximate potential $V(r, T, \mu)$ in (7) for different values of chemical potential and temperature.

In Figure 1, the exact $U(r, T, \mu)$ and the approximate potential $V(r, T, \mu)$ are plotted for different values of chemical potentials. We note that there is a good qualitative agreement between exact potential and approximate potential. In Figure 2, we note a good qualitative agreement between two potentials. By increasing temperature, the positive part of two potentials is reduced. Thus, the present potential gives a good accuracy in comparison with original potential.

In Figure 3, the Debye screening mass is plotted with temperature for different values of chemical potential (a) and also with the chemical potential for different values of temperatures (b). (a) shows that the Debye screening mass decreases with temperature but shifts to upper values by increasing chemical potential. This behavior is in agreement with $[51,52]$. (b) shows that the Debye screening mass increases with the chemical potential but shifts to lower values by increasing temperature in agreement with [43].

3.1. Binding Energy and Heavy-Quarkonium Mass in the NDimensional Space. In this subsection, the binding energy and the heavy-quarkonium mass are calculated such as charmonium and bottomonium mesons in the $\mathrm{N}$-dimensional 

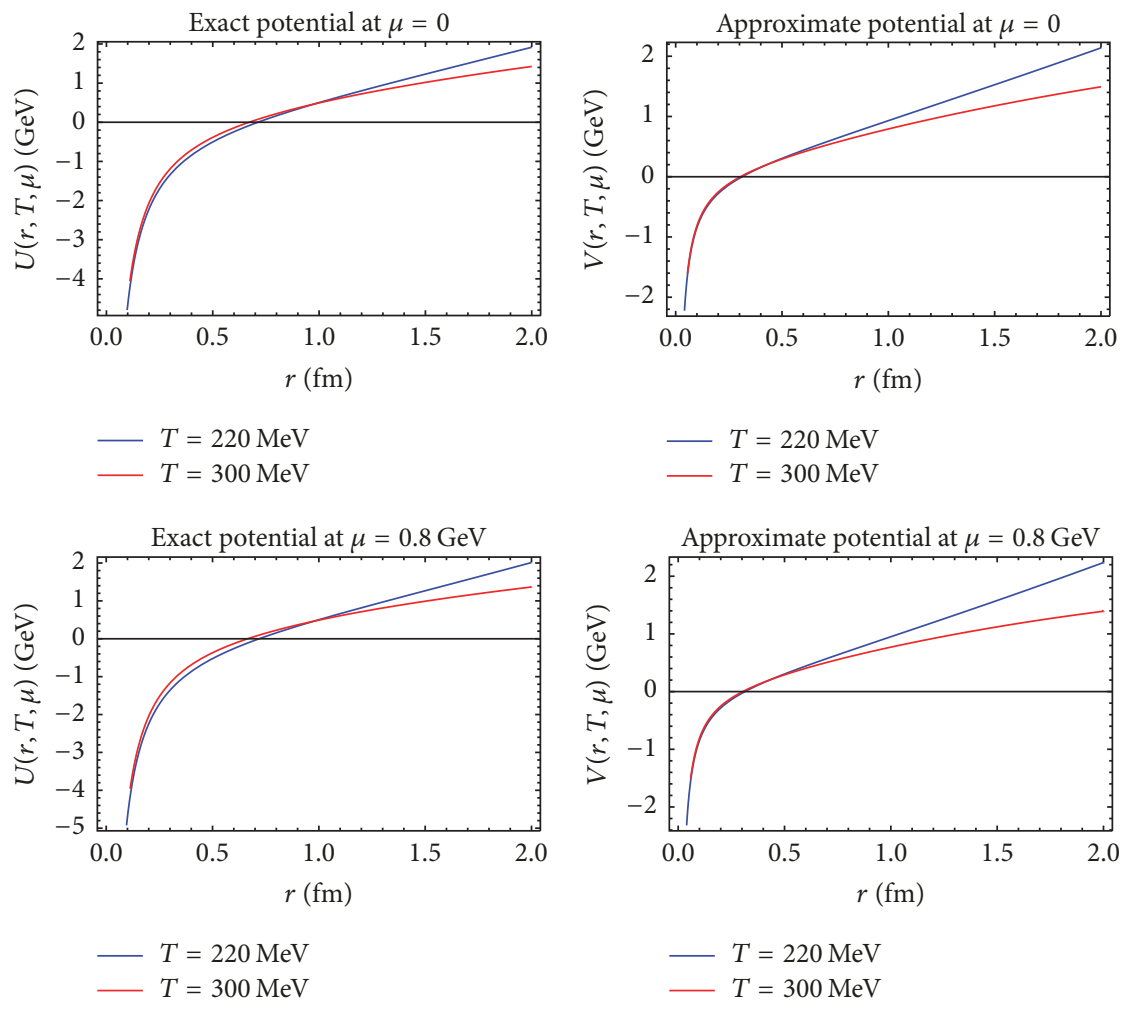

(a)

(b)

FIgure 2: Two potentials are functions of distance $(r)$, for different temperature and chemical potential. The exact potential $U(r, T, \mu)$ in $($ a) and the approximate potential $V(r, T, \mu)$ in (b).
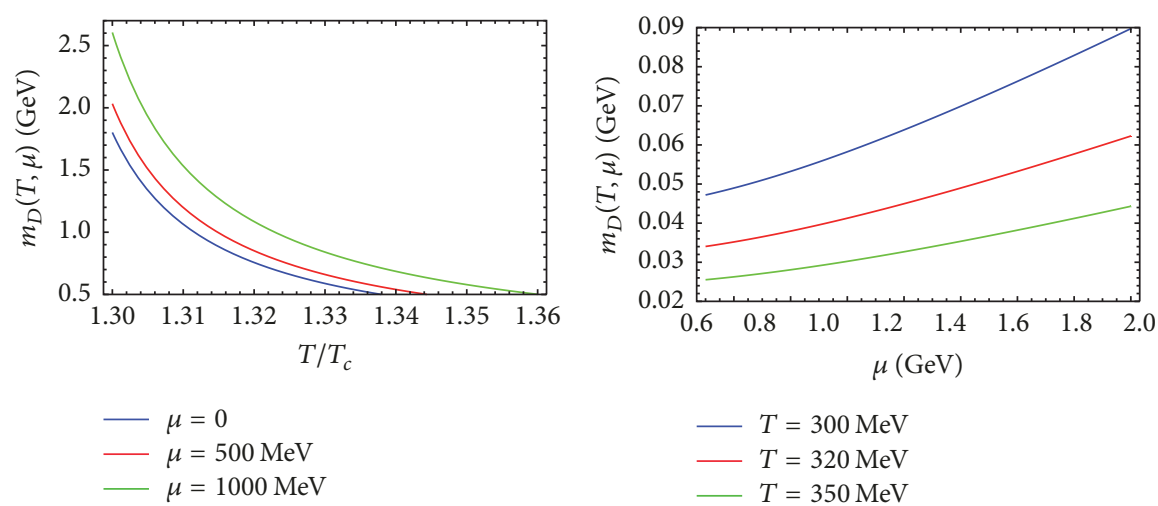

(a)

(b)

FIGURE 3: Debye screening mass with the temperature at different values of chemical potential (a) and Debye screening mass with chemical potential at different values of temperatures (b).

space for any state at finite temperature and chemical potential.

Substituting (8) into (25), therefore the binding energies for the different states of heavy-quarkonium meson at finite temperature and chemical potential take the form

$$
\begin{aligned}
E_{\mathrm{bin}}(T, \mu)= & \alpha_{s} m_{D}(T, \mu) \\
& +\sqrt{\frac{\sigma m_{D}(T, \mu)}{4 \mu_{\mathrm{QQ}}}(2 n+2 l+N)}
\end{aligned}
$$

$$
-\frac{\left(2 \sigma-\alpha_{s} m_{D}^{2}(T, \mu)\right)^{2}}{8 \sigma m_{D}(T, \mu)}
$$

where $\mu_{\mathrm{QQ}}=m_{c} / 2$ for charmonium and $\mu_{\mathrm{QQ}}=m_{b} / 2$ for bottomonium. At zero temperature, we note that Debye mass vanishes. Therefore, energy eigenvalue in (26) is divergent. Thus, (26) is valid only at finite temperature. The behavior of the binding energy for the different states of heavyquarkonium meson is shown in Figures 4, 5, and 6. 


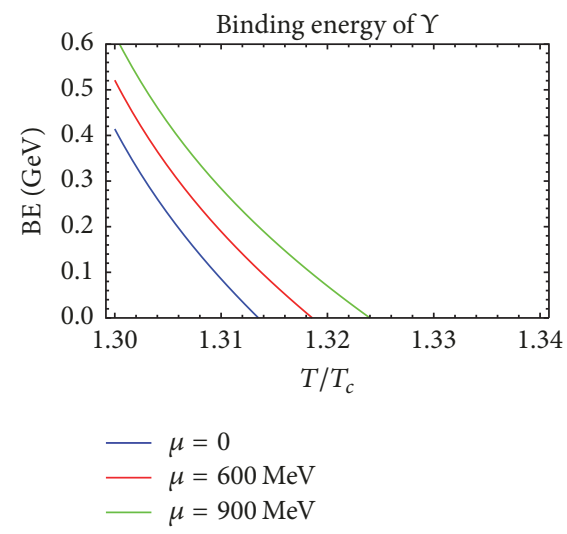

(a)

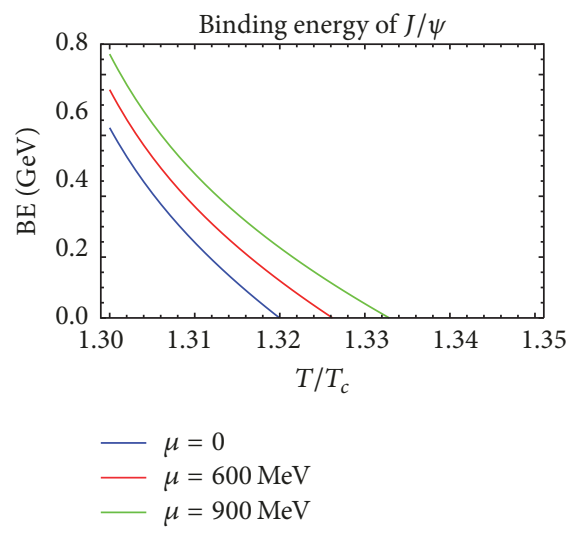

(b)

FIGURE 4: Dependence of $\Upsilon$ binding energy (in $\mathrm{GeV}$ ) on temperature $T / T_{c}$ (a) and dependence of $J / \psi$ binding energy (in $\mathrm{GeV}$ ) on temperature $T / T_{c}$ (b) at different values of chemical potential.

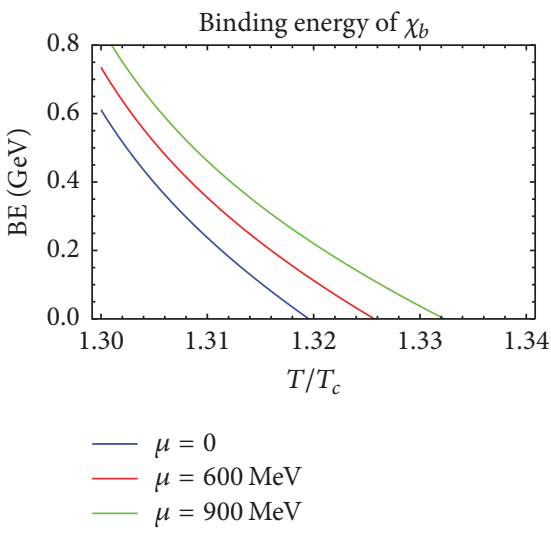

(a)

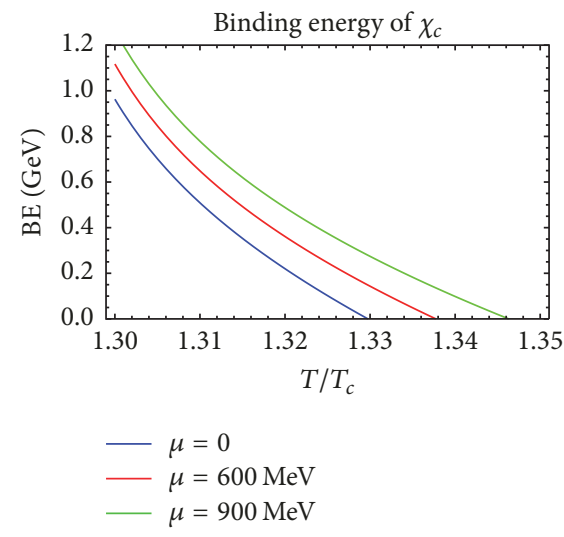

(b)

FIGURE 5: Dependence of $\chi_{b}$ binding energy (in $\mathrm{GeV}$ ) on temperature $T / T_{c}$ (a) and dependence of $\chi_{c}$ binding energy (in $\mathrm{GeV}$ ) on temperature $T / T_{c}$ (b) at different values of chemical potential.

Figures 4 and 5 show the behavior of the binding energy of heavy quarkonia as a function of temperature (in units of $T_{c}$ ) for $1 \mathrm{~S}$ and $1 \mathrm{P}$ states, respectively.

We note that the binding energy becomes weaker with increasing temperature. The dependence of the binding energy on the temperature shows a qualitative agreement with similar results in $[25,29-33]$ and becomes stronger with the chemical potential. Figure 6 shows the dependence of the binding energy of $J / \psi$ and $\Upsilon$ states on the number of dimensions. The binding energy of $J / \psi$ and $\Upsilon$ states increases with the increasing dimensionality number. In Figure 7, the binding energy of charmonium and bottomonium mesons is plotted in the 3-dimensional space. We note that the binding energy increases with increasing finite temperature and chemical potential. Therefore, the effect of finite temperature is stronger than the effect of chemical potential.

Now, for calculating quarkonium mass, we use the following relation [13]:

$$
M=2 m_{\mathrm{Q}}+E_{n l}^{N} .
$$

Substituting (26) into (27), thus the mass spectra for the different states are a function of temperature and chemical potential that takes the following form:

$$
\begin{aligned}
M_{\mathrm{Q}}= & 2 m_{\mathrm{Q}}+\alpha_{s} m_{D}(T, \mu) \\
& +\sqrt{\frac{\sigma m_{D}(T, \mu)}{4 \mu_{\mathrm{QQ}}}}(2 n+2 l+N) \\
& -\frac{\left(2 \sigma-\alpha_{s} m_{D}^{2}(T, \mu)\right)^{2}}{8 \sigma m_{D}(T, \mu)},
\end{aligned}
$$

where $M_{Q}$ is quarkonium mass $Q=(b, c)$ for bottomonium and charmonium.

In Figure 8, quarkonium mass is plotted as a function of temperature for $1 S$ and $1 P$ states, bottomonium in (a) and charmonium in (b). We see that the mass spectra decrease with increasing temperature. The values of $1 \mathrm{P}$ state are larger than the values of $1 S$ state. By increasing chemical potential, the quarkonium mass shifts to larger values. 

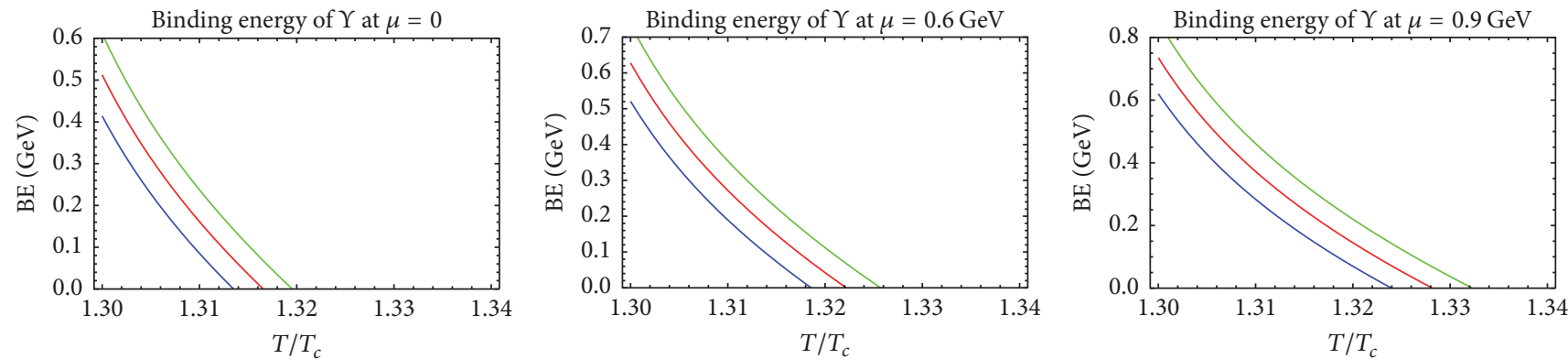

$$
\begin{array}{r}
N=3 \\
-N=4 \\
N=5
\end{array}
$$

$$
\begin{array}{r}
N=3 \\
-N=4 \\
N=5
\end{array}
$$

$$
\begin{aligned}
-N & =3 \\
-N & =4
\end{aligned}
$$

(a)
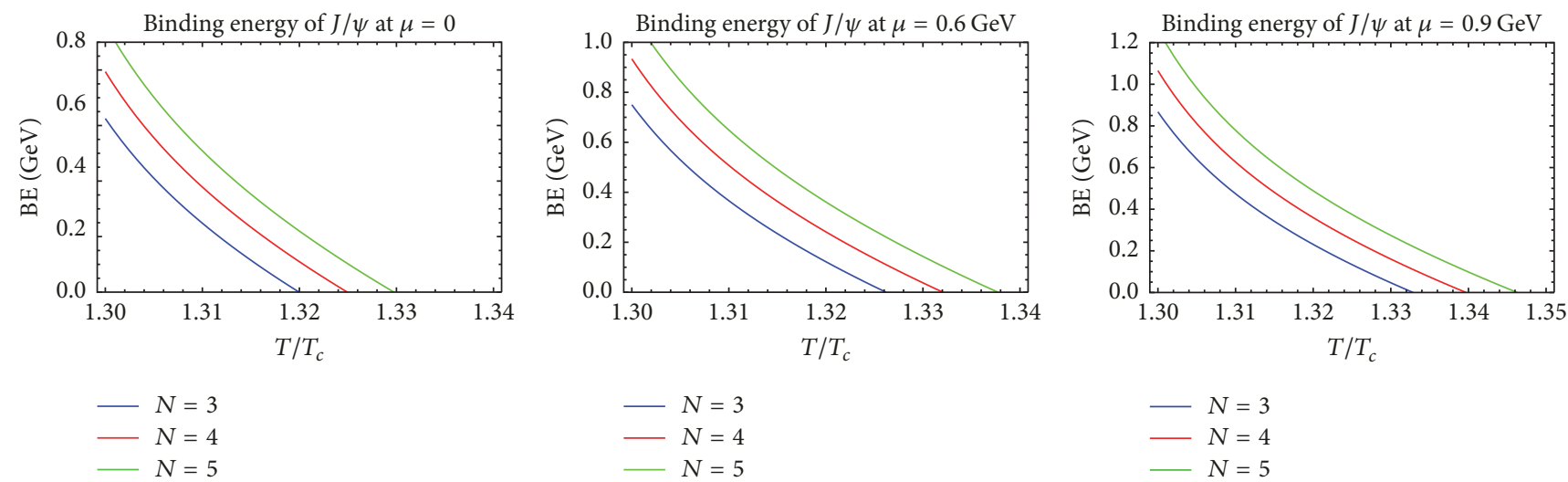

(b)

Figure 6: Dependence of $\Upsilon$ binding energy (in $\mathrm{GeV}$ ) on temperature $T / T_{c}$ (a) and dependence of $J / \psi$ binding energy (in GeV) on temperature $T / T_{c}$ (b) at different values of $N$.

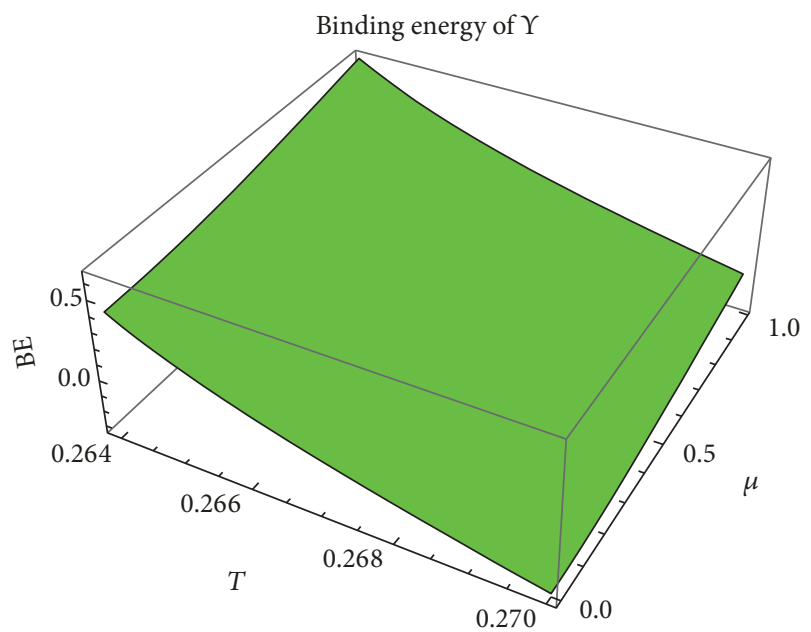

(a)

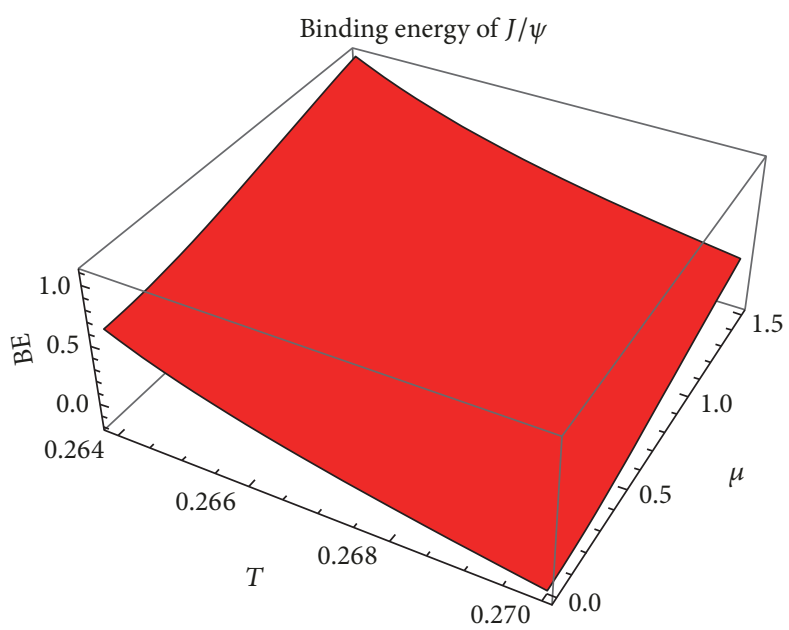

(b)

FIGURE 7: Dependence of $\Upsilon$ binding energy (in $\mathrm{GeV}$ ) on temperature $T / T_{c}$ (a) and dependence of $J / \psi$ binding energy (in $\mathrm{GeV}$ ) on temperature $T / T_{c}(b)$ in 3 dimensions. 
TABLE 1: The dissociation temperature $\left(T_{D}\right)$ with $T_{c}=203 \mathrm{MeV}$ for the quarkonia states (in units of $T_{c}$ ) using $m_{c}=1.6 \mathrm{GeV}$ and $m_{b}=4.7 \mathrm{GeV}$ at $\mu=0$.

\begin{tabular}{lccc}
\hline State & $N=3$ & $N=4$ & $N=5$ \\
\hline$J / \psi$ & $1.31997 T_{c}$ & $1.32493 T_{c}$ & $1.32974 T_{c}$ \\
$\psi^{\prime}$ & $1.32974 T_{c}$ & $1.33441 T_{c}$ & $1.33897 T_{c}$ \\
\hline$Y$ & $1.31351 T_{c}$ & $1.31656 T_{c}$ & $1.31955 T_{c}$ \\
$Y^{\prime}$ & $1.31955 T_{c}$ & $1.32247 T_{c}$ & $1.32534 T_{c}$ \\
\hline
\end{tabular}
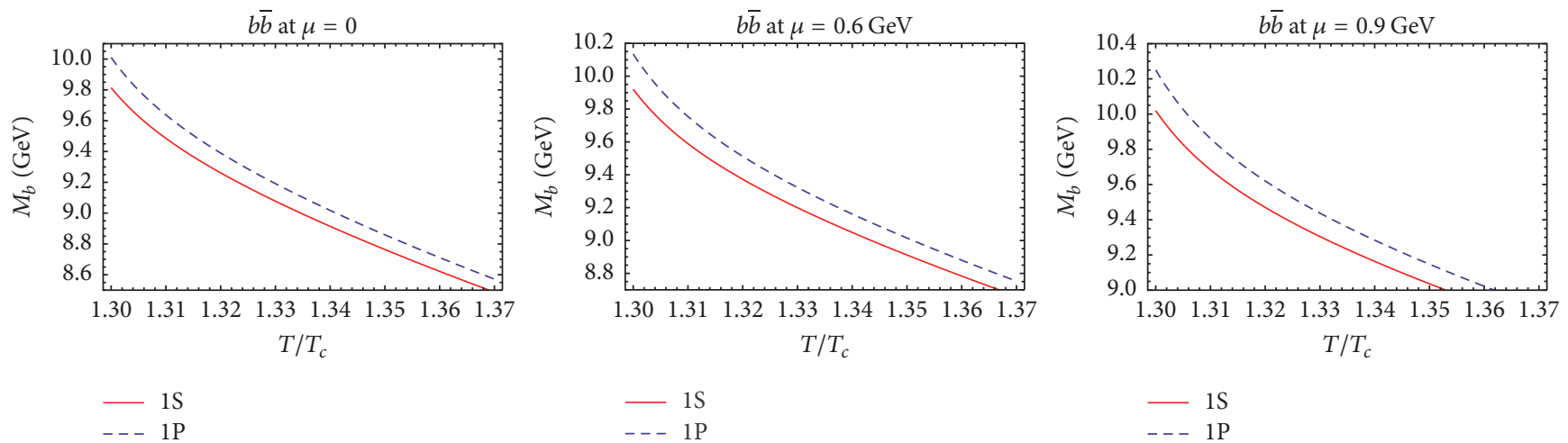

(a)
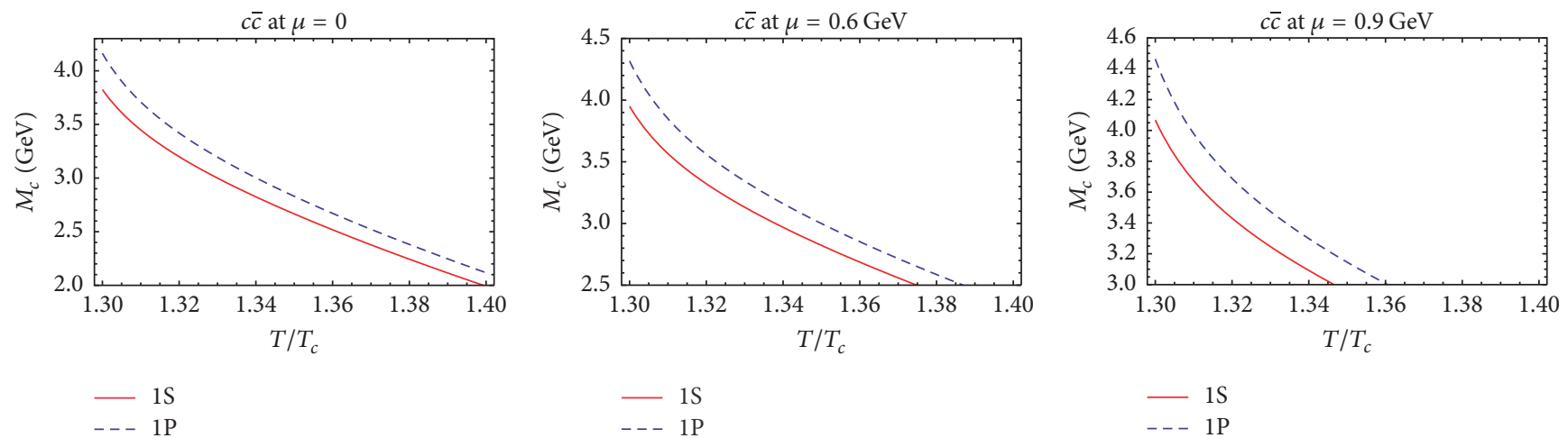

(b)

Figure 8: The mass spectra of heavy quarkonia are plotted as a function of temperature for $1 S$ and $1 P$ states, bottomonium in (a) and charmonium in (b).

In Figures 9 and 10, we study the behavior of the quarkonium mass as a function of temperature (in units of $T_{c}$ ) for $1 \mathrm{~S}$ and $1 \mathrm{P}$ states for different values of chemical potential using two values of quark mass. We noted that the increasing quark mass leads to increasing quarkonium mass as in [28]. In the $1 \mathrm{~S}$ state, the charmonium mass increases from $M_{c}=$ $3.460 \mathrm{GeV}$ to $M_{c}=3.825 \mathrm{GeV}$ at zero chemical potential. At finite chemical potential $(\mu=0.6 \mathrm{GeV})$, charmonium mass increases from $M_{c}=3.588 \mathrm{GeV}$ to $M_{c}=3.950 \mathrm{GeV}$.

3.2. Dissociation Temperature of Heavy Quarkonia in the NDimensional Space. There are a lot of earlier studies for determining the dissociation temperatures for different states of heavy quarkonia. In [24], the authors have calculated the dissociation temperature of the heavy quarkonia from the thermal width $\Gamma(T)$. In [29], authors have put a conservative condition for the dissociation $\Gamma(T)>2 E_{\text {bin }}$. In [30], the authors have calculated the upper bound and the lower bound of the dissociation temperature $\left(T_{D}\right)$ by the condition for the dissociation: $E_{\text {bin }}=T_{D}$ and $E_{\text {bin }}=3 T_{D}$, respectively. In [43], the authors have obtained the dissociation temperature of quarkonia when the binding energies are of the order of the baryon chemical potential.

We calculate the dissociation temperature for different states of heavy quarkonia from the condition $E_{\mathrm{bin}}=0$, since the state is dissociated when its binding energy vanished as in [27].

In Table 1, we have calculated the dissociation temperature for the ground state and the first excited states of $c \bar{c}$ and $b \bar{b}$ at $N=3$ and also at higher dimensional space at $N=$ 4 and $N=5$ when chemical potential vanishes. It is noted from Table 1 that the states dissociate around $1.3 T_{c}$. The values of $J / \psi$ and $\Upsilon^{\prime}$ quantitatively agree with the values recently reported by Agotiya et al. [30]. $\Upsilon$ gives smaller value in 
TABLE 2: The dissociation temperature $T_{D}(\mathrm{MeV})$ at $\mu=0.6 \mathrm{GeV}$ with $T_{c}=185 \mathrm{MeV}$.

\begin{tabular}{lccr}
\hline State & $N=3$ & $N=4$ & $N=5$ \\
\hline$J / \psi$ & $1.45524 T_{c}$ & $1.46167 T_{c}$ & $1.46789 T_{c}$ \\
$\psi^{\prime}$ & $1.46789 T_{c}$ & $1.47393 T_{c}$ & $1.47982 T_{c}$ \\
\hline$\Upsilon$ & $1.44687 T_{c}$ & $1.45082 T_{c}$ & $1.45469 T_{c}$ \\
$Y^{\prime}$ & $1.45469 T_{c}$ & $1.45848 T_{c}$ & $1.46219 T_{c}$ \\
\hline
\end{tabular}
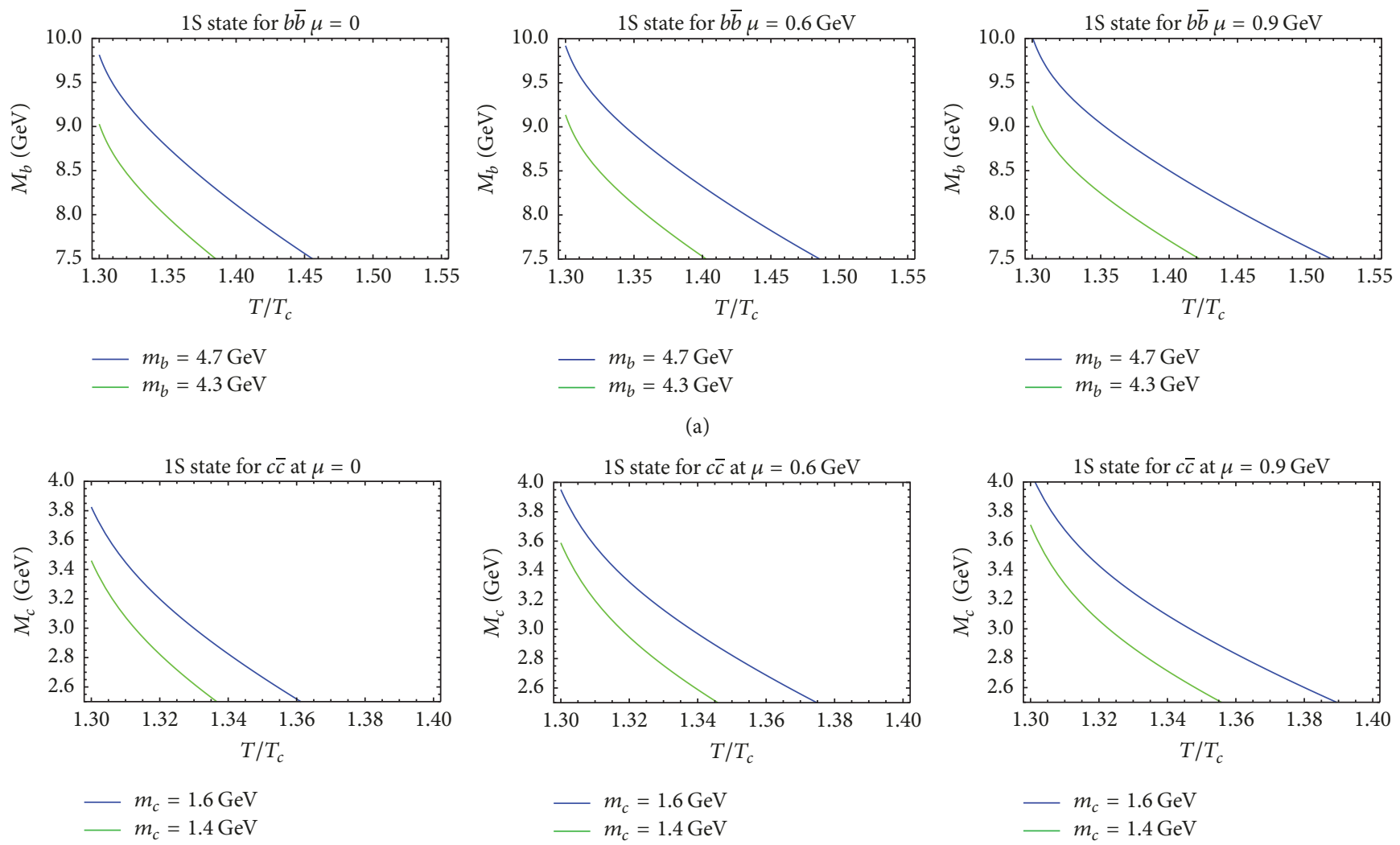

(b)

FIGURE 9: The mass spectra of heavy quarkonia are plotted as a function of temperature for $1 S$ state, bottomonium in (a) and charmonium in (b).

comparison with [30] which equals $1.7 T_{c}$. Also, the value of $\Upsilon$ is in agreement with that in [53] which gives the dissociation temperature of the $1 \mathrm{~S}$ bottomonium

$T_{d}=1.4 T_{c}$. In [30], the dissociation temperature depends on the chosen Debye screening mass. It is important to display the effect of dimensionality number on the dissociation temperature. We note from Table 1 that increasing dimensionality number leads to increasing dissociation temperature at zero chemical potential.

In Table 2, the dissociation temperatures for different states of heavy-quarkonium mesons have been obtained at finite chemical potential $(\mu=600 \mathrm{MeV})$ and the critical temperature $\left(T_{c}=185 \mathrm{MeV}\right)$. One notes that increasing dimensional number leads to a small increase in the dissociation temperatures. In Table 3 , by increasing chemical potential $\mu=900 \mathrm{MeV}$, there is an important observation: an increase in the value of quark chemical potential increases the value of dissociation temperatures. Therefore, the finite chemical and dimensional number play an important role in changing dissociation temperatures which are not taken into account in many previous works such as $[30,53]$.

\section{Summary and Conclusion}

In this paper, we have employed the analytical exact iteration method (AEIM) for determining the analytic solution of the $N$-dimensional radial Schrödinger equation, in which the Cornell potential is generalized at finite temperature and quark chemical potential. The energy eigenvalues have been calculated in the $N$-dimensional space for any state, in which one can obtain the energy eigenvalues in lower dimensions in agreement with recent works. We noted that the energy eigenvalues are only valid for nonzero temperature at any value of chemical potential. The present results are 
TABLE 3: The dissociation temperature $T_{D}(\mathrm{MeV})$ at $\mu=0.9 \mathrm{GeV}$ with $T_{c}=160 \mathrm{MeV}$.

\begin{tabular}{lccr}
\hline State & $N=3$ & $N=4$ & $N=5$ \\
\hline$J / \psi$ & $1.69108 T_{c}$ & $1.69973 T_{c}$ & $1.70811 T_{c}$ \\
$\psi^{\prime}$ & $1.70811 T_{c}$ & $1.71624 T_{c}$ & $1.72417 T_{c}$ \\
\hline$\Upsilon$ & $1.67982 T_{c}$ & $1.68514 T_{c}$ & $1.69035 T_{c}$ \\
$Y^{\prime}$ & $1.69035 T_{c}$ & $1.69545 T_{c}$ & $1.70045 T_{c}$ \\
\hline
\end{tabular}
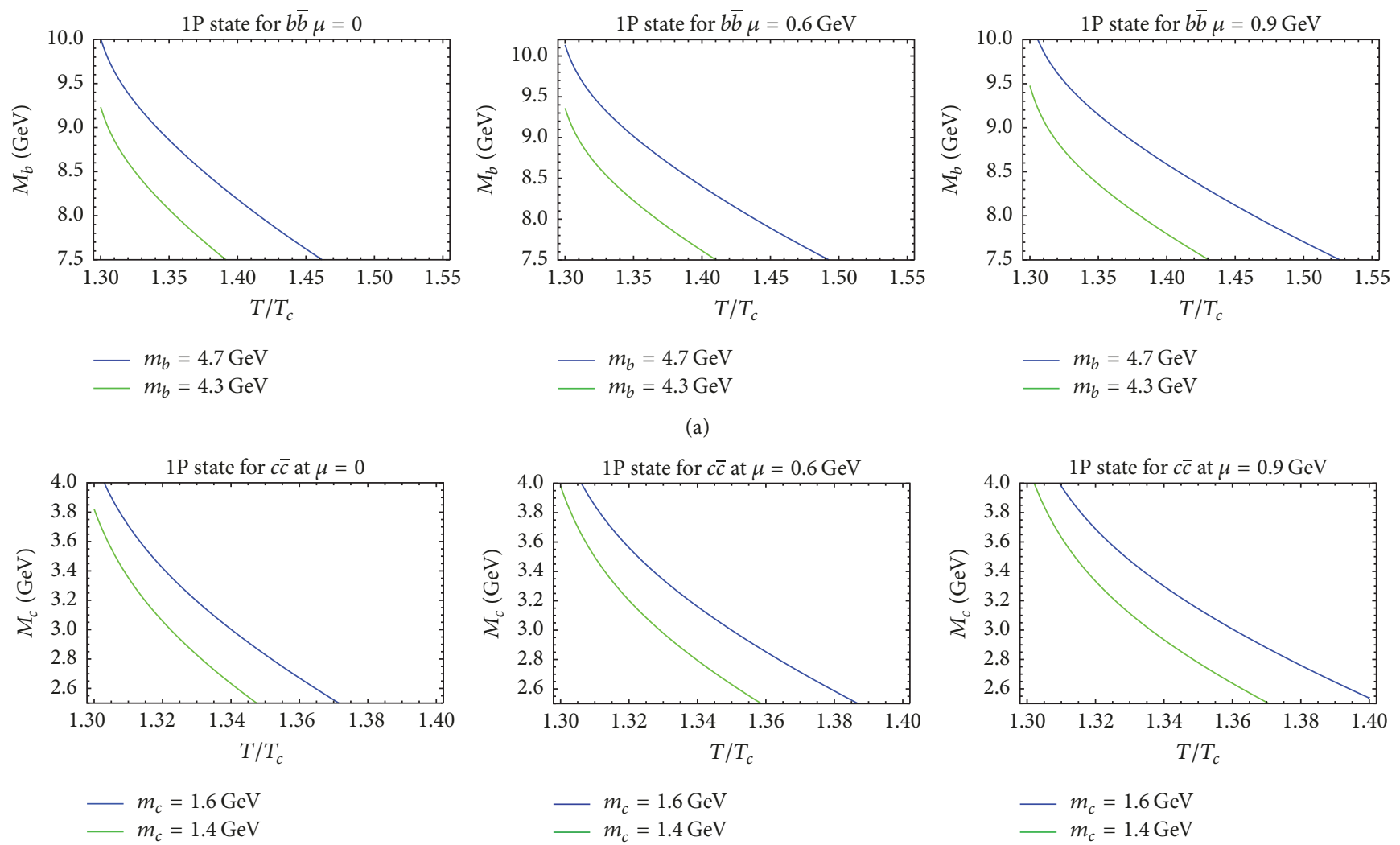

(b)

FIGURE 10: The mass spectra of heavy quarkonia are plotted as a function of temperature for $1 P$ state, bottomonium in (a) and charmonium in (b).

applied to studying properties of heavy quarkonia such as charmonium and bottomonium. The effect of temperature, chemical potential, and dimensionality number is studied on the binding energies and the mass spectra of heavy quarkonia. The present results are in agreement with recent works $[25,29,33]$. The binding energies of $1 S$ and $1 \mathrm{P}$ states for charmonium and bottomonium have been studied in comparison with other studies [11,30]. Additionally, the effect of the dimensionality number $(N)$ on the values of dissociation temperatures of heavy quarkonia has been studied at zero and finite chemical potential. We consider the effect of finite quark chemical potential on quarkonium properties which play an important role in QGP and the studied values of the chemical potential are never reached in the heavy-ion collision. We conclude that the present potential with using AEIM is successful in describing the quarkonium properties at hot and dense mediums from normal dimensional space to higher dimensional space. We hope to extend this work by including external magnetic field and hyperfine interactions which need more investigations as a future work.

\section{Conflicts of Interest}

The authors declare that there are no conflicts of interest regarding the publication of this paper.

\section{References}

[1] J. J. Sakurai, Modern Quantum Mechanics, Addison-Wesley Publishing, New York, NY, USA, 1967.

[2] A. Sommerfeld, Wave-Mechanics, London, UK, 1930.

[3] D. J. Griffiths, Introduction to Quantum Mechanics, Pearson Prentice Hall, Upper Saddle River, NJ, USA, 2nd edition, 2005.

[4] R. P. Feynman and A. R. Hibbs, Quantum Mechanics and Path Integrals, McGrawHill, New York, NY, USA, 1965. 
[5] E. Z. Liverts, E. G. Drukarev, R. Krivec, and V. B. Mandelzweig, "Analytic presentation of a solution of the Schrodinger equation," Few-Body Systems, vol. 44, p. 367, 2008.

[6] R. De, R. Dutt, and U. Sukhatme, "Mapping of shape invariant potentials under point canonical transformations," Journal of Physics A: Mathematical and General, vol. 25, p. L843, 1992.

[7] R. N. Choudhury and M. Mondal, "Hill determinant method with a variational parameter," Physical Review A, vol. 40, p. 6080, 1989.

[8] R. Kumar and F. Chand, "Series solutions to the N-dimensional radial Schrödinger equation for the quark-antiquark interaction potential," Physica Scripta, vol. 85, no. 5, Article ID 055008, 2012.

[9] A. N. Ikot, O. A. Awoga, and A. D. Antia, "Bound state solutions of d-dimensional Schrödinger equation with Eckart potential plus modified deformed Hylleraas potential," Chinese Physics B, vol. 22, Article ID 020304, 2013.

[10] D. Agboola, “The Hulthén potential in D-dimensions," Physica Scripta, vol. 80, no. 6, Article ID 065304, 2009.

[11] H. Hassanabadi, S. Zarrinkamar, and A. Rajabi, "Exact solutions of $D$-dimensional schrödinger equation for an energydependent potential by NU method," Communications in Theoretical Physics, vol. 55, p. 541, 2011.

[12] M. Abu-Shady, "Heavy quarkonia and Bc-mesons in the Cornell potential with harmonic oscillator potential in the $\mathrm{N}$-dimensional Schrödinger Equation," International Journal Applied Mathematicsts and Theortical Physics, vol. 2, p. 16, 2016.

[13] R. Kumar and F. Chand, "Asymptotic Study to the $N$ Dimensional Radial Schrödinger Equation for the QuarkAntiquark System," Communications in Theoretical Physics, vol. 59, pp. 528-532, 2013.

[14] T. Das, "Treatment of $N$-dimensional Schrödinger equation for anharmonic potential via Laplace transform," Electronic Journal of Theoretical Physics, vol. 13, p. 207, 2016.

[15] G. Chen, "Exact solutions of the $N$-dimensional radial Schrodinger equation with the coulomb potential via Laplace transform," Zeitschrift für Naturforschung, vol. 59a, p. 875, 2004.

[16] R. N. Chaudhuri and M. Mondal, "Eigenvalues of anharmonic oscillators and the perturbed Coulomb problem in $\mathrm{N}$ dimensional space," Physical Review A, vol. 52, p. 1850, 1995.

[17] H. Hassanabadi, B. H. Yazarloo, S. Zarrinkamar, and M. Solaimani, "Approximate analytical versus numerical solutions of Schrödinger equation under molecular Hua potential," International Journal of Quantum Chemistry, vol. 112, p. 3706, 2012.

[18] H. Rahimov, H. Nikoofard, S. Zarrinkamar, and H. Hassanabadi, "Approximate arbitrary-state solutions of Dirac equation for modified deformed Hylleraas and modified Eckart potentials by the NU method," Applied Mathematics and Computation, vol. 219, p. 4710, 2013.

[19] E. M. Khokha, M. Abu-Shady, and T. A. Abdel-Karim, "Quarkonium masses in the $N$-dimensional space using the analytical exact iteration method," International Journal of Theoretical and Applied Mathematics, vol. 2, p. 86, 2016.

[20] K. J. Oyewumi, F. O. Akinpelu, and A. D. Agboola, "Exactly complete solutions of the pseudoharmonic potential in $\mathrm{N}$ dimensions," International Journal of Theoretical Physics, vol. 47, p. 1039, 2008.

[21] S. Ikhdair and R. Sever, "Polynomial solutions of the Mie-type potential in the D-dimensional Schrodinger equation," Journal of Molecular Structure, vol. 855, p. 13, 2008.

[22] S. Ikhdair and R. Sever, "Exact solutions of the modified Kratzer potential plus ring-shaped potential in the $D$-dimensional
Schrodinger equation by the Nikiforov-Uvarov method," International Journal of Modern Physics C, vol. 19, p. 221, 2008.

[23] S. M. Kuchin and N. V. Maksimenko, "Theoretical estimations of the spin - averaged mass spectra of heavy quarkonia and Bc mesons," Universal Journal of Physics and Application, vol. 7, p. 295, 2013.

[24] D. Kharzeev, L. McLerranand, and H. Satz, "Non-perturbative quarkonium dissociation in hadronic matter," Physics Letters B, vol. 356, p. 349, 1995.

[25] D. Blaschke, O. Kaczmarek, E. Laermann, and V. Yudichev, "Heavy quark potential and quarkonia dissociation rates," The European Physical Journal C, vol. 5, Article ID 02327, 2005.

[26] H. Satz, "Colour deconfinement and quarkonium binding," Journal of Physics G: Nuclear and Particle Physics, vol. 32, p. 25, 2006.

[27] H. Satz, "Charm and Beauty in a Hot Environment," BI-TP 2006/06, 2006, https://arxiv.org/abs/hep-ph/0602245.

[28] W. M. Alberico, A. Beraudo, A. De Pace, and A. Molinari, "Quarkonia in the deconfined phase: Effective potentials and lattice correlators," Physical Review D, vol. 75, Article ID 074009, 2007.

[29] A. Mocsy and P. Petreczky, "Color screening melts quarkonium," Physical Review Letter, vol. 99, Article ID 211602, 2007.

[30] V. Agotiya, V. Chandra, and B. K. Patra, "Dissociation of quarkonium in a hot QCD medium: Modification of the interquark potential," Physical Review C, vol. 80, Article ID 025210, 2009.

[31] L. Thakur, N. Haque, U. Kakade, and B. K. Patra, "Dissociation of quarkonium in an anisotropic hot QCD medium," Physical Review D, vol. 88, Article ID 054022, 2013.

[32] S. Chao-Yi, Z. Jia-Qing, M. Zhi-Lei, and L. Yun-De, "Thermal Width for Heavy Quarkonium in the Static Limit," Chinese Physics Letters, vol. 32, no. 12, Article ID 121201, 2015.

[33] L. Thakur and B. K. Patra, "Quarkonium dissociation in an anisotropic QGP," Journal of Physics: Conference Series, vol. 668, Article ID 012085, 2016.

[34] V. Kumar, V. Chandra, M. Y. Jamal, and I. Nilima, "Dissociation of heavy quarkonium in hot QCD medium in a quasiparticle model," Physical Review D, vol. 94, Article ID 094006, 2016.

[35] H. Vija and M. H. Thoma, "Braaten-Pisarski method at finite chemical potential," Physics Letters B, vol. 342, no. 1-4, pp. 212218, 1995.

[36] A. Peshier, B. Kämpfer, and G. Soff, "Equation of state of deconfined matter at finite chemical potential in a quasiparticle description," Physical Review C, vol. 61, Article ID 045203, 2000.

[37] M. Döring, S. Ejiri, O. Kaczmarek, F. Karsch, and E. Laermann, "Screening of heavy quark free energies at finite temperature and non-zero baryon chemical potential," The European Physical Journal C, vol. 46, p. 179, 2006.

[38] H. Gervais and S. Jeon, "Photon production from a quark-gluon plasma at finite baryon chemical potential," Physical Review C, vol. 86, Article ID 034904, 2012.

[39] A. Monnai, "Dissipative hydrodynamic effects on baryon stopping," Physical Review C, vol. 86, Article ID 014908, 2012.

[40] S. S. Singh, "Free energy and direct photon emission at finite chemical potential," Journal of Physics: Conference Series, vol. 535, Article ID 012002, 2014.

[41] V. S. Filinov, M. Bonitz, Y. B. Ivanov, E.-M. Ilgenfritz, and V. E. Fortov, "Thermodynamics of the quark-gluon plasma at finite chemical potential: Color Path integral Monte Carlo results," Contributions to Plasma Physics, vol. 55, p. 203, 2015. 
[42] S. M. Sanches Jr., D. A. Fogaca, F. S. Navarra, and H. Marrochio, "Cavitation in a quark gluon plasma with finite chemical potential and several transport coefficients," Physical Review C, vol. 92, Article ID 025204, 2015.

[43] U. Kakade and B. K. Patra, "Quarkonium dissociation at finite chemical potential," Physical Review C, vol. 92, Article ID 024901, 2015.

[44] R. Kumar and F. Chand, "Energy Spectra of the Coulomb Perturbed Potential in N-Dimensional Hilbert Space," Chinese Physics Letters, vol. 29, Article ID 060306, 2012.

[45] F. Karsch, M. T. Mehr, and H. Satz, "Color screening and deconfinement for bound states of heavy quarks," Zeitschrift für Physik C Particles and Fields, vol. 37, p. 617, 1988.

[46] B. Liu and Y. B. dong, "Quark binding potential and debye screening length," Communications in Theoretical Physics, vol. 26, p. 425, 1996.

[47] R. A. Schneider, QCD Phenomenology at High Temperatures [Dissertation], Techn. University, München, Germany, 2002.

[48] A. O. Barut, M. Berrondo, and G. Garcia-Calderon, "Narrow resonances as an eigenvalue problem and applications to high energy magnetic resonances: An exactly soluble model," Journal of Mathematical Physics, vol. 21, p. 1851, 1980.

[49] S. özcelik and M. Simsek, "Exact solutions of the radial Schrödinger equation for inverse-power potentials," Physics Letters A, vol. 152, p. 145, 1991.

[50] S. M. Ikhdair and M. Hamzavi, "Spectral properties of quantum dots influenced by a confining potential model," Physica B, vol. 407, p. 4797, 2012.

[51] Y. Burnier and A. Rothkopf, "A gauge invariant Debye mass and the complex heavy-quark potential," Physics Letters B, vol. 753, p. 232, 2016.

[52] O. Kaczmarek and F. Zantow, "Static quark-antiquark interactions in zero and finite temperature QCD: I. heavy quark free energies, running coupling, and quarkonium binding," Physical Review D, vol. 71, Article ID 114510, 2005.

[53] J. I. Kapusta, "Equation of state and phase fluctuations near the chiral critical point," Physical Review C, vol. 81, Article ID 055201, 2010. 

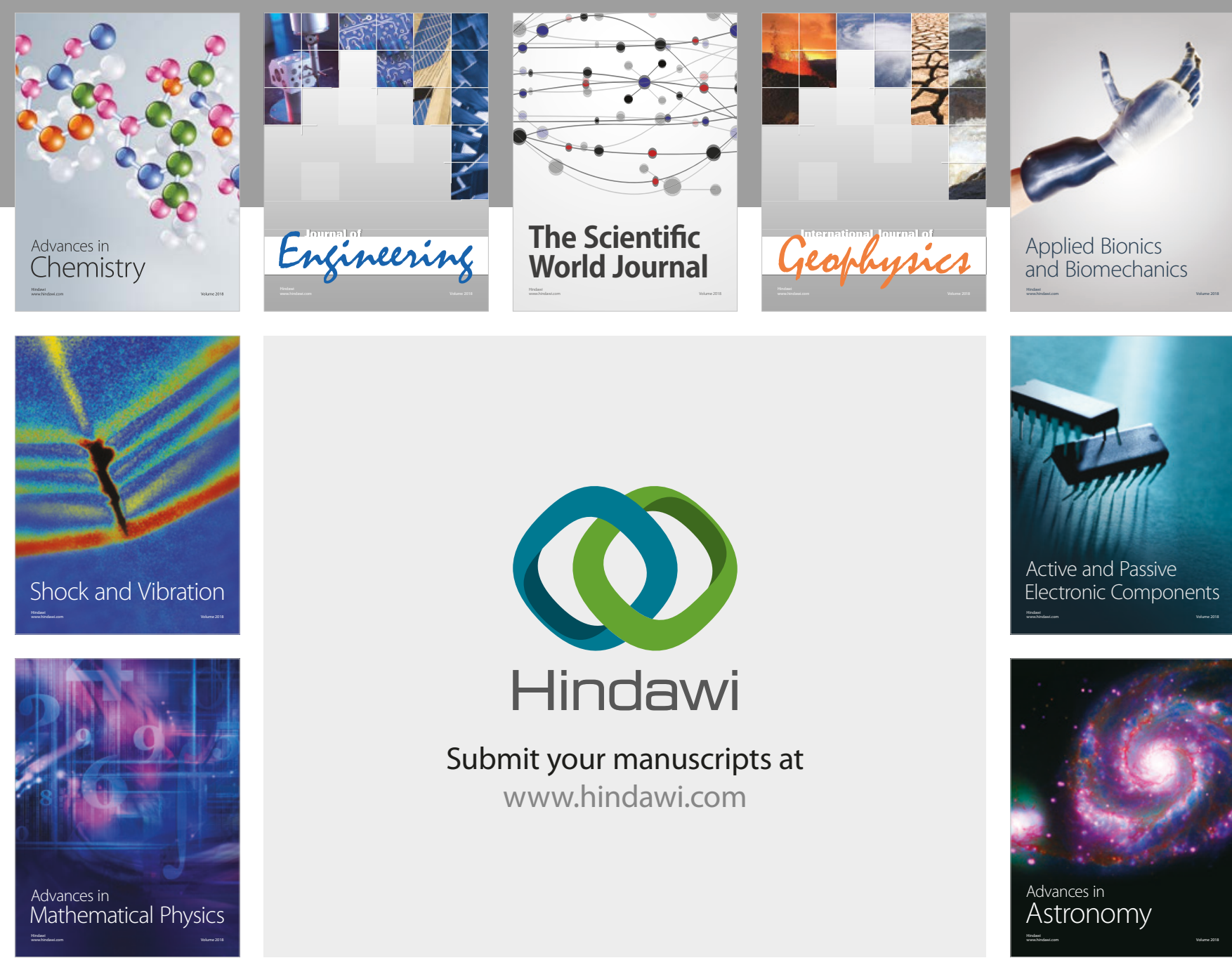

Submit your manuscripts at

www.hindawi.com

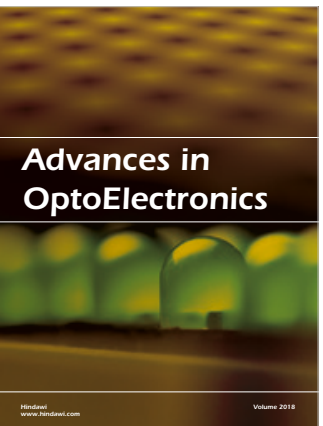

\section{Rotcting Machinery}
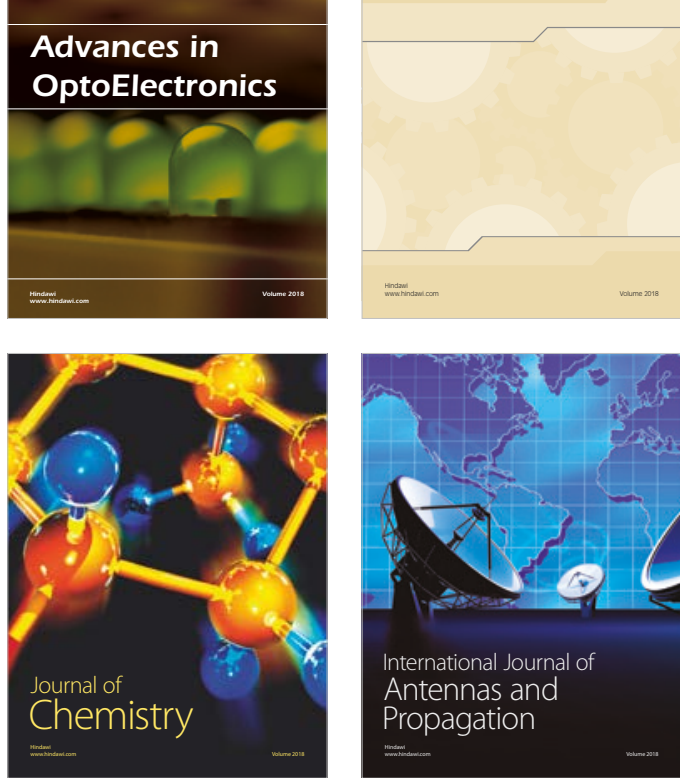

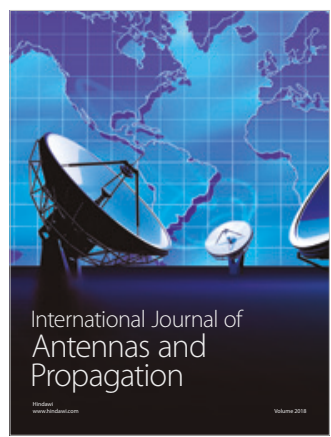

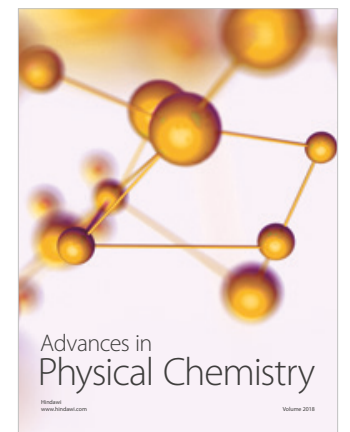

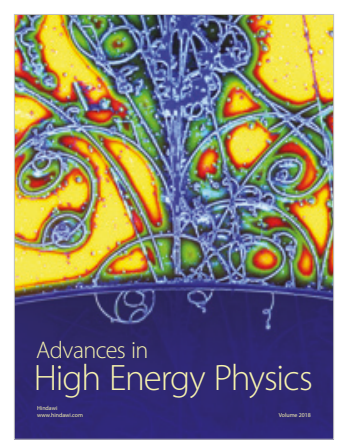

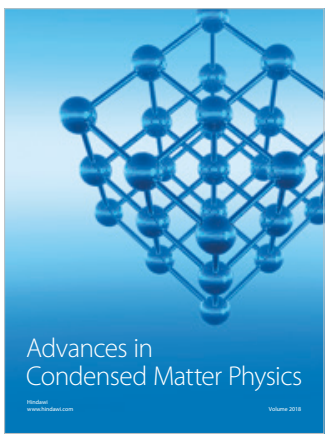

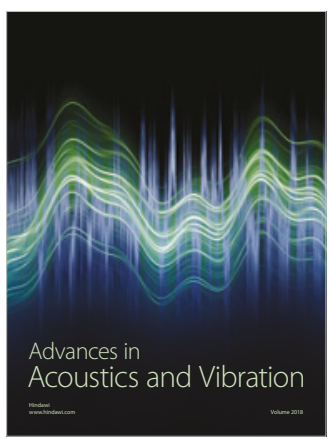

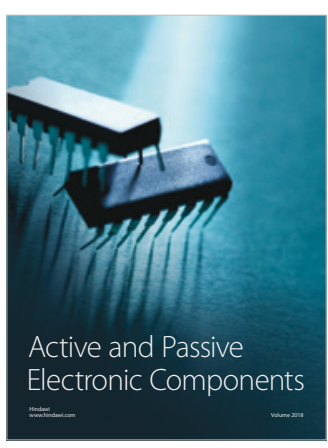
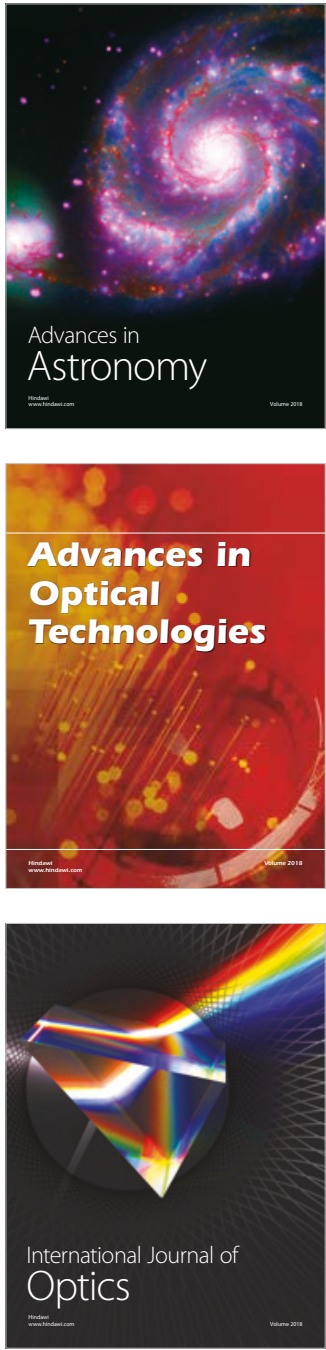